\title{
Boosting postnatal oligodendrogenesis in a mouse model of Multiple Sclerosis
}

Joana Moura Mateus $^{* 1,2}$, Marta Alonso Gomes ${ }^{1,2}$, Rita Soares ${ }^{1,2}$, Sara L. Paulo ${ }^{1,2}$, Ângelo F. Chora ${ }^{2}$, Ana M. Sebastião ${ }^{1,2}$, Sara Xapelli ${ }^{1,2}$

${ }^{1}$ Instituto de Farmacologia e Neurociências, Faculdade de Medicina, Universidade de Lisboa, Lisboa, Portugal; ${ }^{2}$ Instituto de Medicina Molecular-João Lobo Antunes (iMM), Faculdade de Medicina, Universidade de Lisboa, Lisboa, Portugal *joana.mateus@medicina.ulisboa.pt

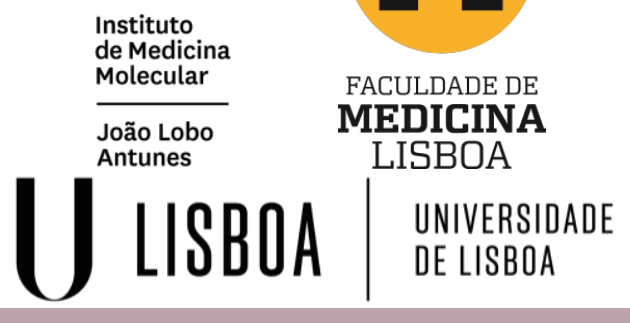

\section{Background}

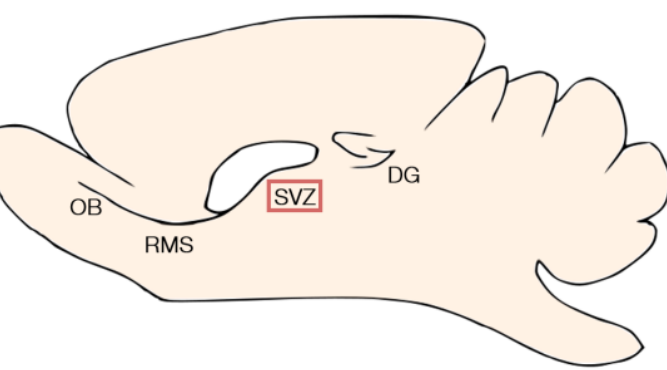

Subventricular Zone (SVZ) - germinal niche present in the adult mammalian brain ${ }^{1}$.

SVZ-derived oligodendrocyte precursor cells (OPCs) migrate to lesioned areas to form mature myelinating (OLs) $)^{2,3}$.

Adenosine (ADO) receptor signalling pathways - involved in the modulation of neuroinflammation in $\mathrm{MS}^{4,5}$.

$\mathbf{A}_{2 \mathrm{~A}} \mathbf{R}$ are expressed in all cells in CNS and present high affinity for ADO. Previous results

- $A_{2 A} R$ activation promotes an increase in the number of oligodendrocytes derived from SVZ neural stem cells in vitro ${ }^{6}$.

$A_{2 A} R$ activation inhibits the proliferation and migration of OPCs derived from the SVZ?.

Aim Study the role of $A_{2 A} R$ in adult oligodendrogenesis derived from SVZ-NSCs in the EAE model of MS

Results

\section{EAE model characterization}

Motor impairment is proportional to EAE clinical score

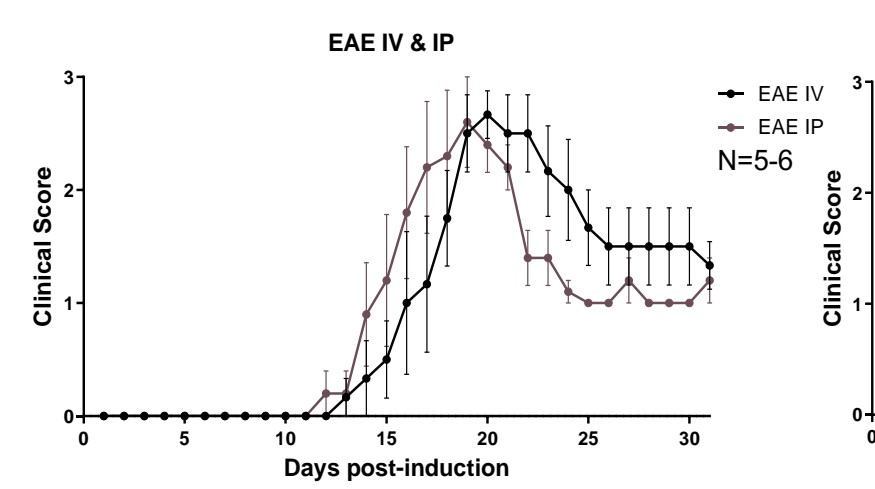

Rotarod

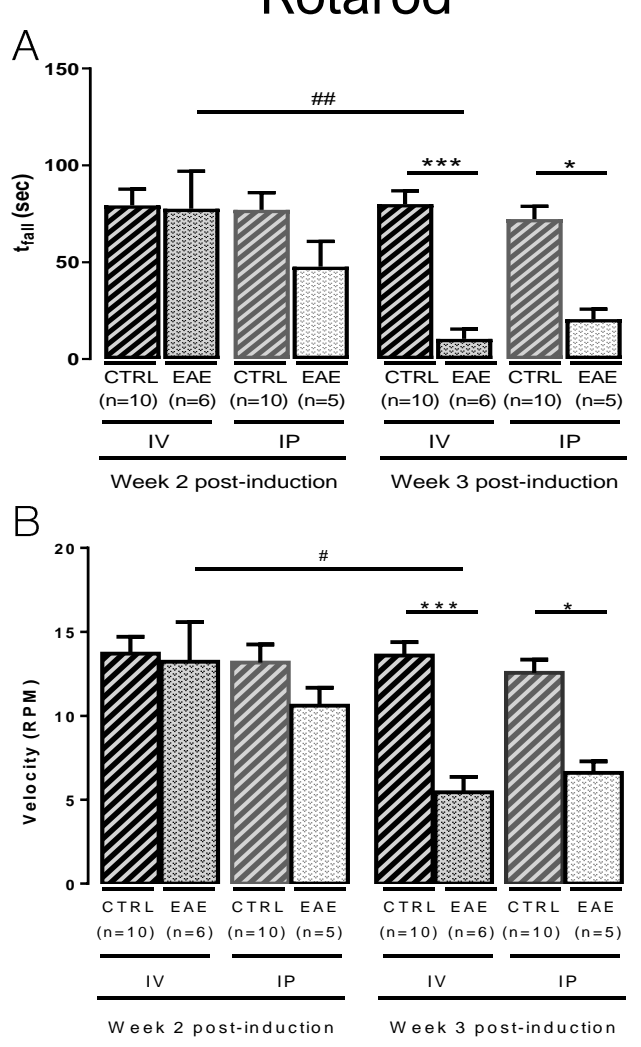

EAE SPF mice tend to have increased inflammatory cytokine levels
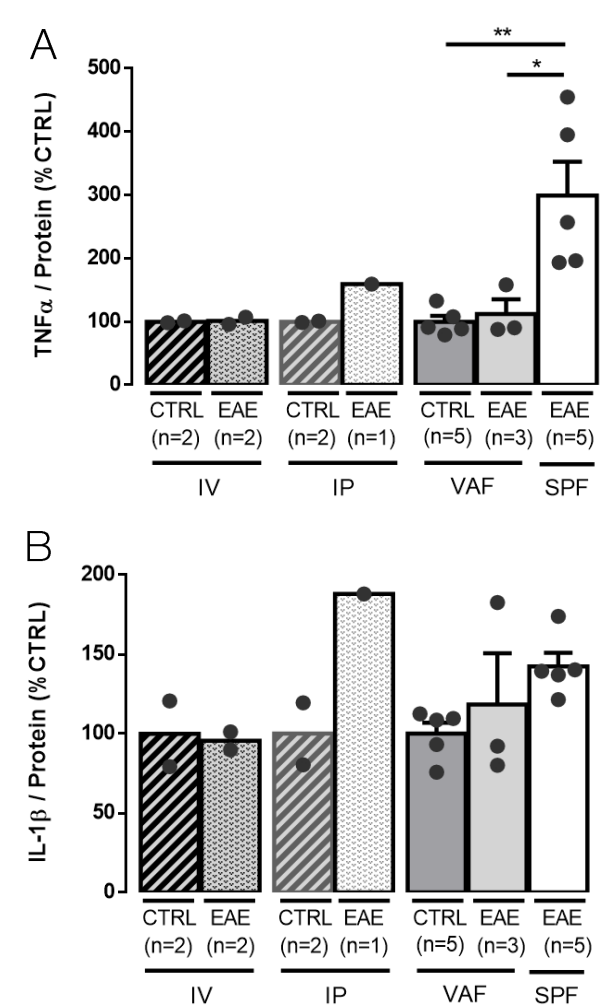

EAE VAF \& SPF

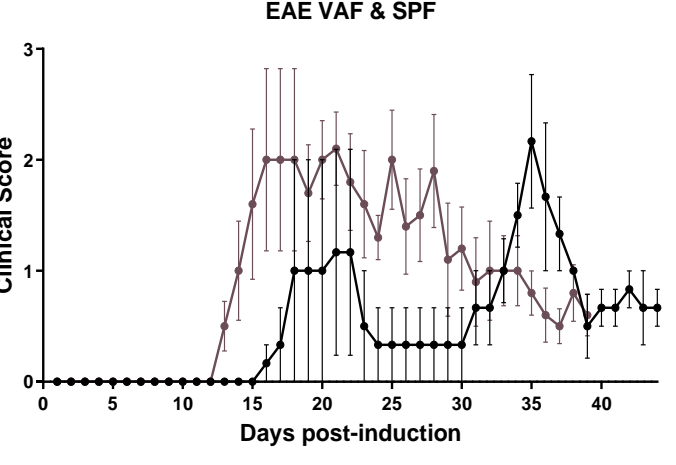

Pole test

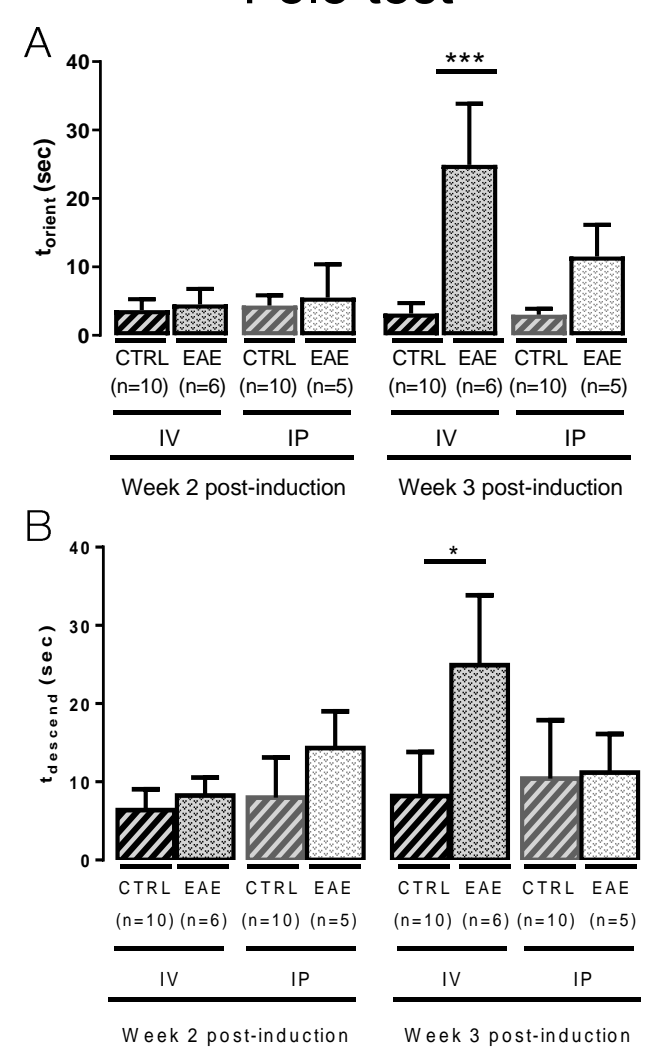

$\mathrm{BrdU}^{+} / \mathrm{NG}^{+}$cells are significantly increased in the CC of EAE mice

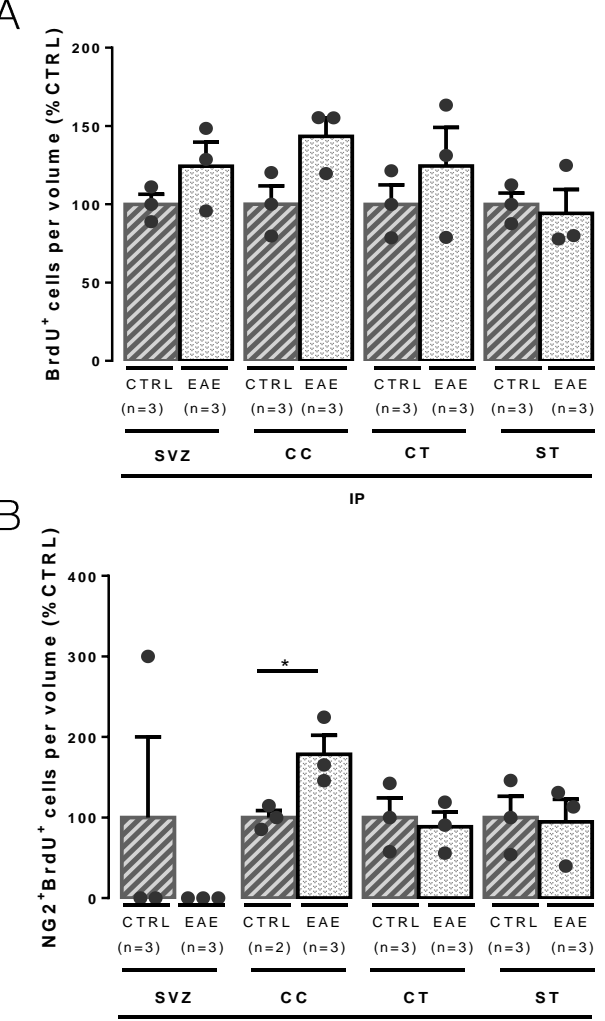

Methods

EAE model characterization

BrdU administration

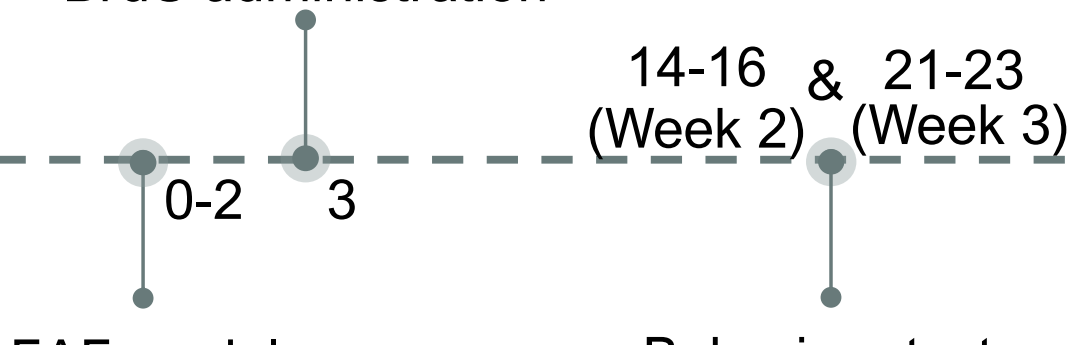

EAE model

Behaviour tests

Adenosine $\mathrm{A}_{2 \mathrm{~A}} \mathrm{R}$ modulation $+\mathrm{C} 57 \mathrm{BL} / 6$
$8-10$ weeks old

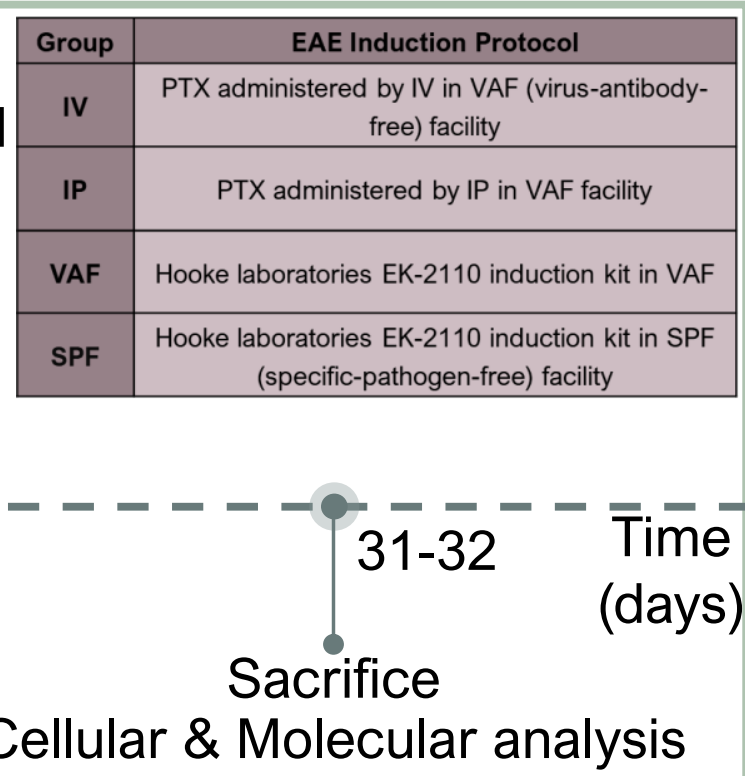

Cellular \& Molecular analysis induction

\section{ime} Adenosine $A$ RA modulation

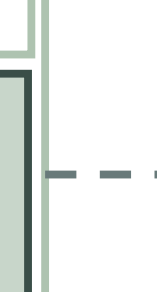

EAE model induction

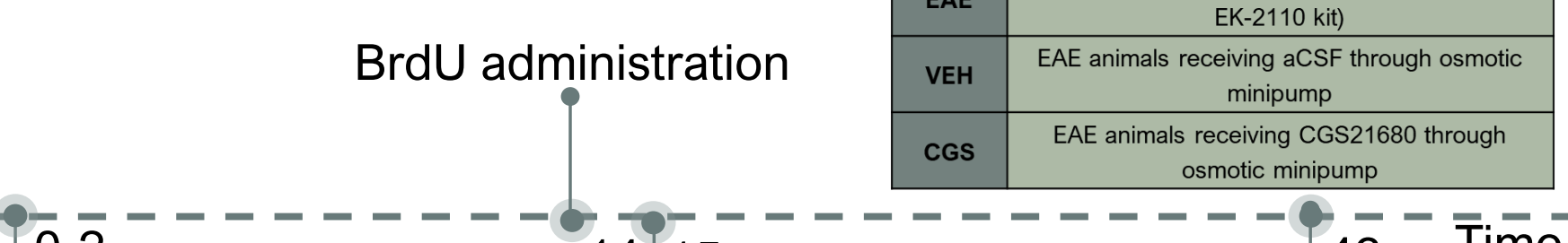

BrdU administration
Osmotic minipump implantation for $A_{2 A} R$ agonist administration [CGS 21680] - $100 \mathrm{nM}$

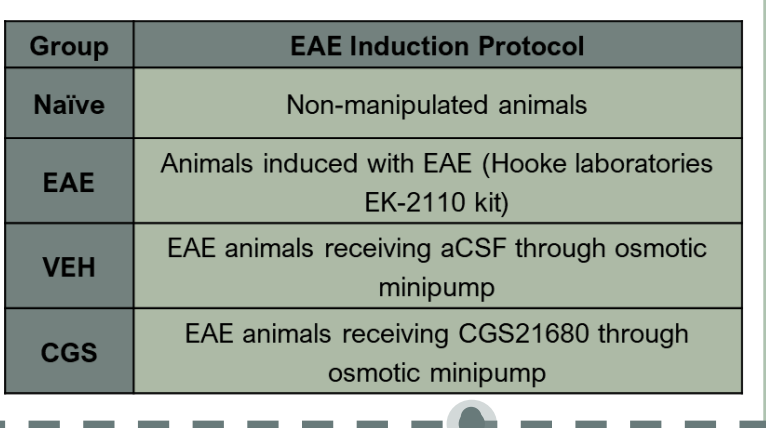

43 Time (days)

Sacrifice

Cellular \& Molecular analysis

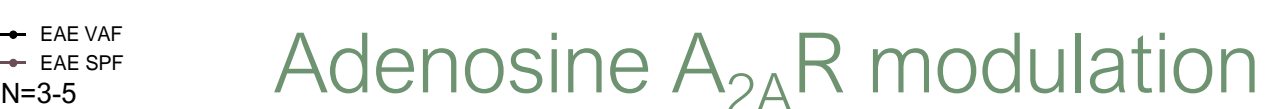
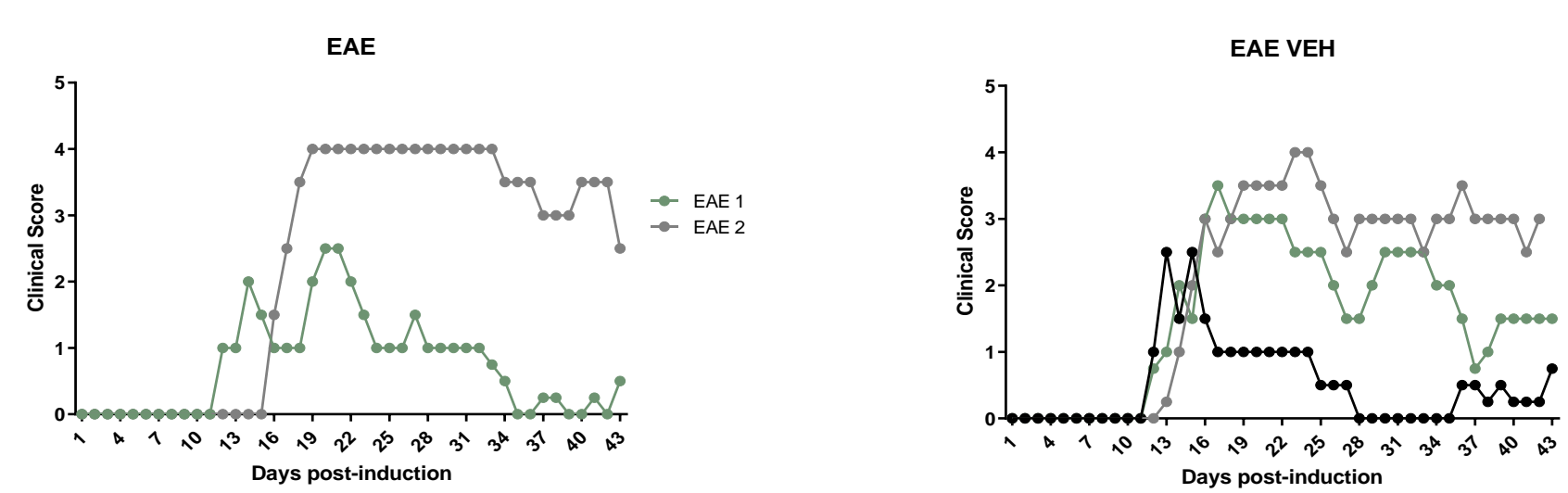

$\rightarrow$ EAE VEH 1
$\rightarrow$ EAE VEH 2
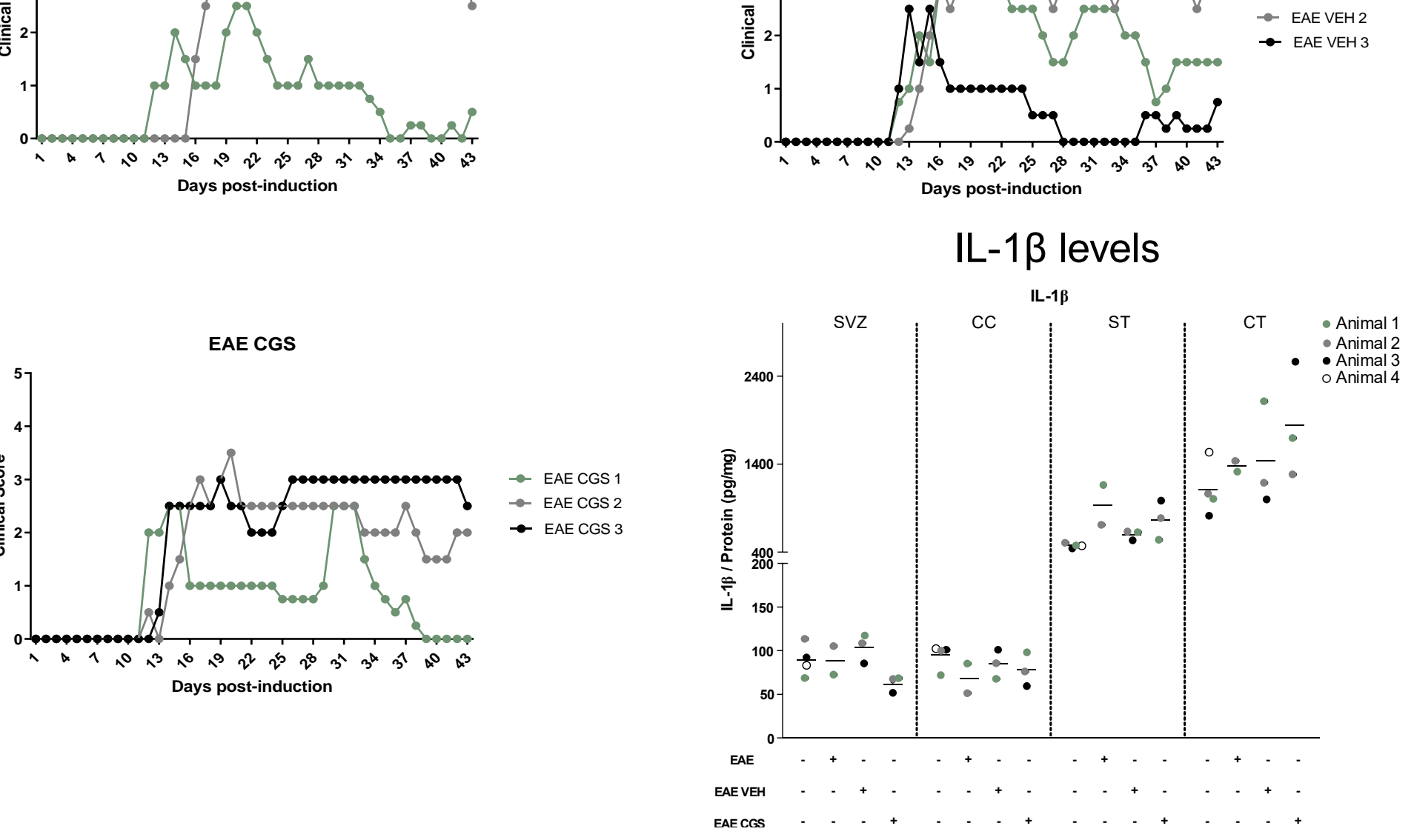

\section{Conclusions}

Clinical scores, behaviour tests and molecular analysis allowed for a characterization of EAE phenotype progression.

Under EAE conditions, there is a migration of SVZ-derived OPCs into the $\mathrm{CC}$, one of the affected regions.

Ongoing work regarding $A_{2 A} R$ modulation entails the evaluation of adult oligodendrogenesis by $\mathrm{IHC}$.

Statistical significance was determined using unpaired two-tailed Students' t-test or Kruskal-Wallis test with Dunn's correction for multiple comparisons, whether there were two or more conditions to test, respectively. ${ }^{*} p<0,05 ;{ }^{* *} p<0,01 ;{ }^{* * *} p<0,001 ; \# p<0,05 ; \# p<0,01 ; \# p<0,001$.

References: 1- Alvarez-Buylla A., García-Verdugo J. M. \& Tramontin A. D., Nat. Rev. Neurosci. 2, 287-93 (2001); 2- Picard-Riera N. et al., Proc. Natl. Acad. Sci. U.S.A. 99, 13211-16 (2002); 3- Menn B. et al., J Neurosci 26, 7907-18 (2006); 4- Vincenzi F. et al., Eur. J. Immunol. 43, 2206-16 (2013); 5- Mills J. H. et al., J. Immunol. 188, 5713-22 (2012); 6- d'Armada Moreira, A., Master thesis, 2015; 7- Afonso, C., Master thesis, 2017. 